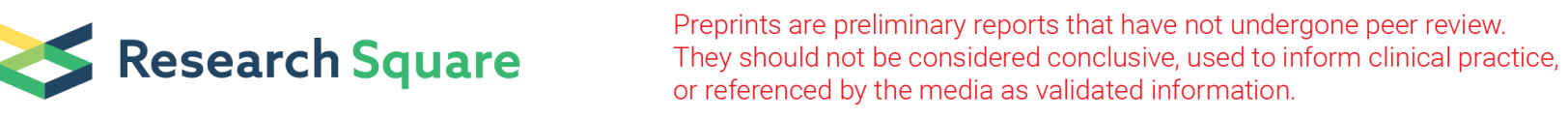

\title{
Improving 1-propanol Force Field: a New Methodology
}

José Abundio Daniel Alva-Tamayo

Universidad de Guadalajara

Iván Guillén-Escamilla

Universidad de Guadalajara

Gloria Arlette Méndez-Maldonado

Universidad de Guadalajara

José Guillermo Méndez-Bermúdez ( $\sim$ jose.bermudez@academicos.udg.mx )

Universidad de Guadalajara https://orcid.org/0000-0001-5276-8909

\section{Research Article}

Keywords: force field, parameterization, molecular dynamics, 1-propanol, structure factor, solubility

Posted Date: November 9th, 2021

DOI: https://doi.org/10.21203/rs.3.rs-947062/v1

License: (c) (1) This work is licensed under a Creative Commons Attribution 4.0 International License. Read Full License 


\title{
Improving 1-propanol force field: a new
}

\author{
methodology
}

\author{
José Abundio Daniel Alva-Tamayo ${ }^{1}$, Iván \\ Guillén-Escamilla ${ }^{1}$, Gloria Arlette Méndez-Maldonado ${ }^{2}$ \\ and José Guillermo Méndez-Bermúdez ${ }^{1^{*}}$
}

${ }^{1 *}$ Centro Universitario de los Valles, Universidad de Guadalajara, Carretera Guadalajara-Ameca Km. 45.5, Ameca, 46600, Jalisco, México.

${ }^{2}$ Centro Universitario de Ciencias Exactas e Ingenierías, Universidad de Guadalajara, Blvd. Marcelino García Barragán No. 1412, Guadalajara, 44430, Jalisco, México.

\author{
*Corresponding author(s). E-mail(s): \\ jose.bermudez@academicos.udg.mx; \\ Contributing authors: daniel.alva@alumno.udg.mx; \\ ivan.guillen@valles.udg.mx; \\ gloria.mmaldonado@academicos.udg.mx;
}

\begin{abstract}
A new force field for 1-propanol, in the united and all atom models, has been obtained by combining two different empirical methodologies. The first was developed by scaling atom charges, and Lennard-Jones parameters to fit the dielectric constant, surface tension, and density ((2018) J. Chem. Theory Comput. 14:5949-5958). The second methodology consists of moving these parameters and together with the bond distance to obtain the liquid-vapor phase diagram of the $\mathrm{CO}_{2}$ molecule ((1995) J. Phys. Chem. 99:12021-12024). The last methodology is used to obtain the self-diffusion coefficient, which was not considered in the first one. With this new methodology, the experimental density, dielectric constant, surface tension, and self-diffusion coefficient at ambient temperature could be achieved. Furthermore, we show the temperature dependence of the aforementioned properties. The static structure factors are in accordance
\end{abstract}


with the experimental spectrum. Solubility is increased to the experimental value for the united atom model after applying this methodology and for all atom scheme, the experimental solubility value is maintained.

Keywords: force field, parameterization, molecular dynamics, 1-propanol, structure factor, solubility

\section{Introduction}

Alcohols are the most used solvents in science and engineering, with 1-propanol being the water-miscible compound with the largest aliphatic chain. Reproducing most of the thermodynamic, structural, and dynamic properties of any molecule is one of the main goals in molecular simulation. Many force fields reproduce some of these properties, mainly conformational energies, density, and $\Delta H_{\mathrm{V}}$ are reproduced using the OPLS/AA force field [1]; $\Delta H_{\mathrm{V}}, \Delta G_{\mathrm{solv}}$, and density are the target properties for the GROMOS force field [2]; free energies and many conformational, bulk, and interface properties in biological macromolecules are estimated from CHARMM [3]; and conformational energies and root-mean-square displacement to build molecular structures of proteins and nucleic acids for organic molecules are predicted by GAFF [4].

The parameterization methods have been applied to reproduce the properties that current force fields do not consider. Cole et al. [5] used quantum mechanics to obtain partial charges and Lennard-Jones potential parameters (LJPP) to model organic and Biomolecular systems, including proteins. One hundred forty-six organic liquids are benchmarked in the OPLS/AA, CGenFF, and GAFF force fields by Caleman et al. [6]. A methodology, know as three steps systematic parameterization procedure (3SSPP) was proposed to develop force fields $[7,8]$, where intermolecular parameters were used to fit some target properties, such as the charge molecule distribution, the energy, and distance 
LJPP for obtaining dielectric constant, surface tension, and density, respectively. Recently, a methodology has been developed to improve the 3SSPP to force fields by assigning the Hirshfeld electronic density charge distribution in some polar liquids to obtain the experimental self-diffusion coefficient [14]. New force fields for SPC [9] and TIP4P/2005 [10] water models were developed by considering that there is a dipole moment of minimum density at a fixed temperature to match the dielectric constant at room temperature and the temperature of maximum density.

On the other hand, Ríos-López et al. [11] parameterized tensoactives by scaling the distance and energy LJPP to obtain the micelle radius and surface tension, respectively. Different force field models have been proposed by modifying the force field of semi-flexible $\mathrm{CO}_{2}$ molecules, such as scaling the charge and LJPP [12], and by changing the angle force constant and bond length [13], to obtain thermodynamics properties and the liquid-vapor coexistence curve.

With alcohols, a four-site methanol force field was developed by MartínezJiménez et al. [15]. This united atom (UA) model has an extra site to match the dipole moment for methanol so the temperature dependence of dielectric constant and surface tension fit experimental data. It was proposed for mixtures, a linear scaling for the geometrical combination rule, for both mixtures, methanol oxygen with water oxygen and propanol methyl group with water oxygen, to improve the density, dielectric constant, $\Delta V$, and $\Delta H$ for different molar methanol concentrations.

In the case of 1-propanol in the all atom (AA) scheme, a force field focuses on modifying only the partial charges of the hydroxyl functional group, thereby achieving self-diffusion, which is close to the experimental value [16]. However, the obtained dielectric constant is lower than the experimental value after 
parameterizing (Table 1). Meanwhile, for UA model, there was a parameterization for 1-propanol force field [17] using the 3SSPP methodology [7]. The obtained results are listed in Table 1.

Recently, Zangi [18] combined the OPLS/AA force field to model hydroxyl groups for alcohols and L-OPLS for the hydrocarbon tails to overcome the high attraction and prevent liquid-solid phase transition at ambient temperature. Although bulk density and enthalpy of vaporization were calculated, this force field did not improve self-diffusion coefficients; they were over or under predicted values for most of the studied alcohol molecules.

Finally, a new parameterization method, four steps systematic parametrization procedure (4SSPP), was developed by García-Melgarejo et al. [8]. The 4SSPP fitted the solubility, self-diffusion coefficient, surface tension, and dielectric constant for 1-propanol and other compounds in the TraPPE-UA force field. This methodology improves the 3SSPP force field [7] where solubility and self-diffusion coefficient are close to experimental values. However, this methodology has not been tested for the AA scheme. The OPLS/AA 1propanol force field is water-soluble before applying parameterization, see Fig. 5, so the 4SSPP methodology cannot be applied to this force field.

The rest of the work is summarized as follows: the computational details are described in Section II, and the new methodology to obtain the 1-propanol force fields is described in Section III. Then, the results obtained and discussions are given in Section IV. Finally, conclusions are made in Section V.

\section{Computational details}

We developed molecular dynamics simulations [19] in GROMACS software version 5 [20]. The NPT ensemble at constant $T=298.15 \mathrm{~K}$ and $P=1$ 
atm was used to calculate the density, dielectric constant, and self-diffusion coefficient. The NVT ensemble was used to obtain surface tension. Nosé-Hoover thermostat $\left(\tau_{T}=0.5 \mathrm{ps}\right)$ and Parrinello-Rahman barostat $\left(\tau_{P}=1 \mathrm{ps}\right)$ were used to keep temperature and pressure constant, respectively. In addition, a time step of $\Delta t=0.002 \mathrm{ps}$ and periodic boundary conditions were considered. A cutoff radius of $1.2 \mathrm{~nm}$ and $2.5 \mathrm{~nm}$ for the bulk and liquid-vapor was used, respectively. LINCS constraints and PME were established.

A system with 500 1-propanol molecules in the NPT ensemble for the OPLS/AA [1] and UA [17] force fields was built to obtain the density, dielectric constant, and self-diffusion coefficient. LigParGen software [1] was used to get the force field and molecule configuration in the AA scheme. An NVT system with 2000 molecules in a box of $5.3 \mathrm{~nm} \times 5.3 \mathrm{~nm} \times 15.000 \mathrm{~nm}$ was created to obtain the surface tension, a reciprocal grid of $0.12 \mathrm{~nm}$ in the $x$ and $y$-directions and $0.294 \mathrm{~nm}$ in $z$-direction was set in NVT simulations.

Solubility is obtained in the NPAT ensemble, this liquid-liquid simulations were carried out at constant molecules (4000 water molecules and 1000 alcohols), where temperature and pressure were set at $298 \mathrm{~K}$ and 1 bar, respectively, and the surface area was equal to $A=L_{x} \times L_{y}=5.1 \times 5.1 \mathrm{~nm}^{2}$ of dimension. For both, NVT and NPAT ensembles the truncation distance was $2.5 \mathrm{~nm}$, and long-range corrections for energy and pressure were not considered.

All simulations in the NPT ensemble were running up to $200 \mathrm{~ns}$, and in the last 5 ns the density, self-diffusion constant, and dielectric constant were obtained. In addition, surface tension and solubility were calculated with 5 ns after $10 \mathrm{~ns}$ of equilibration. 


\section{Methodology}

A molecular dynamic simulation was executed to obtain the aforementioned target properties, the obtained results are listed in Table 1. The original OPLS/AA force field (column 3) gave a high percentage error $(>5 \%)$ for the dielectric constant and self-diffusion coefficient, and low percentage error $(<5 \%)$ for surface tension. In addition, the percentage error in density was $<2 \%$ because this force field was obtained by adjusting this property. The OPLS/AA force field was compared with a modified OPLS/AA force field [16], where the self-diffusion coefficient has a percentage error of $11.8 \%-1.6 \%$, with respect to three experimental data, and the dielectric constant has a percentage error of $28 \%$. This model showed a dielectric constant with a high percentage error with no surface tension value. The parameterized UA 1-propanol is a modified force field that improves the original force field [17] by applying the 3SSPP methodology [7], as observed from the last column of Table 1. This methodology involves the following procedure:

Table 1 Estimated dielectric constant, density, self-diffusion coefficient, and surface tension from 1-propanol in the OPLS/AA force field, a modified OPLS/AA developed by T. Kulschewski et. al, and a parameterized UA force field with 3SSPP methodology. Percentage errors provided in parenthesis are concerned with experimental values.

\begin{tabular}{|c|c|c|c|c|c|}
\hline Property & Experiment & Ref. & $\begin{array}{r}\text { OPLS /AA } \\
(\% \text { error) }\end{array}$ & $\begin{array}{l}\text { Modified OPLS/AA } \\
\text { Ref. [16] (\% error) }\end{array}$ & $\begin{array}{r}\text { 3SSPP UA } \\
\text { Ref. [17] (\% error) }\end{array}$ \\
\hline $\bar{\epsilon}$ & $\begin{array}{l}20.1 \\
20.4\end{array}$ & $\begin{array}{l}{[21]} \\
{[17]}\end{array}$ & $\begin{array}{r}13.8(31.3) \\
(32.4)\end{array}$ & $\begin{array}{r}14.5(27.9) \\
(27.6)\end{array}$ & $\begin{array}{r}19.98(0.6) \\
(2.1)\end{array}$ \\
\hline$\rho\left(k g / m^{3}\right)$ & $\begin{array}{l}800.21 \\
803.40\end{array}$ & $\begin{array}{l}{[22]} \\
{[23]}\end{array}$ & $\begin{array}{r}815.0(1.8) \\
(1.4)\end{array}$ & $\begin{array}{r}798.9(0.2) \\
(0.6)\end{array}$ & $\begin{array}{r}797.39(0.4) \\
(0.7)\end{array}$ \\
\hline$D\left(10^{-9} \mathrm{~m}^{3} / \mathrm{s}^{2}\right)$ & $\begin{array}{l}0.552 \\
0.590 \\
0.627\end{array}$ & $\begin{array}{l}{[24]} \\
{[25]} \\
{[26]}\end{array}$ & $\begin{array}{r}0.9228(67.2) \\
(56.4) \\
(47.2)\end{array}$ & 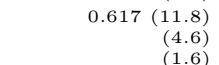 & $\begin{array}{r}0.298(46.0) \\
(49.5) \\
(52.5)\end{array}$ \\
\hline$\gamma(m N / m)$ & 23.26 & {$[27]$} & $24.6(5.8)$ & - & $23.3(0.2)$ \\
\hline
\end{tabular}

1) All partial charges of the molecule are scaled to obtain the dielectric constant in the NPT ensemble.

2) The distance LJPP, $\sigma$, is modified to obtain the surface tension in the NVT ensemble.

3) In the NPT ensemble, the energy LJPP, $\epsilon$, is scaled to obtain the density 
of the system.

4) Finally, we repeat the 1) - 3) steps, to obtain $<5 \%$ deviations from experimental values.

We observed in the 3SSPP methodology that the dielectric constant, density, and surface tension have percentage errors $<5 \%$, but the self-diffusion coefficient has a high percentage error, see [7] and Table 1.

On the contrary, Harris and Yung [13], modified the LJPP and reduced the bond distance by $1 \%$, to improve the $\mathrm{CO}_{2}$ force field. Therefore, we used the bond reduction and the 3SSPP methodology to formulate a new parameterization called 3SSPP/bond which is summarized below:

1) The 3SSPP methodology is applied to a force field.

2) After, bond distance is reduced to increase the self-diffusion.

3) Finally, the 3SSPP methodology is applied again to obtain experimental values with $5 \%$ or less percentage error.

This new methodology is applied to 1-propanol force fields. Step 2, of the proposed methodology, increases the self-diffusion coefficient, see Table 2, the second column has the goal properties obtained by applying the 3SSPP methodology to the 1-propanol original AA force field (step 1 of the new methodology). The reduction of bond distance for the molecule monotonically increases the density and self-diffusion coefficient. On the contrary, the dielectric constant and surface tension reach their maximum value for a bond length of $-2 \%$. After, Step 3 is applied to this force field to obtain the experimental values. It can be noted that the surface tension and dielectric constant are high, with respect to experimental values, after reducing the bond distance, 
making sure that both properties are kept close to their experimental values after step 3. In the case of density, the bond distance's reduction increases the molecular mobility and self-diffusion coefficient. That means, after parameterizing, the density, dielectric constant, and surface tension values approach the experimental values. The results are shown in the next section.

Table 2 Estimated values of dielectric constant, density, self-diffusion coefficient, and surface tension after $1 \%, 2 \%$, and $3 \%$ reduction in bond distance of 1 -propanol molecule in the OPLS/AA parameterized force field. The maximum values obtained for surface tension and dielectric constant are highlighted at the $-2 \%$ reduction in bond distance.

\begin{tabular}{crrrr}
\hline \hline Property & OPLS/AA 3SSPP & $-1 \%$ & $-2 \%$ & $-3 \%$ \\
\hline \hline$\epsilon$ & 22.23015 & 20.97 & $\mathbf{2 1 . 7 8 6 8}$ & 20.8618 \\
$\rho\left(\mathrm{kg} / \mathrm{m}^{3}\right)$ & 802.075 & 813.711 & 826.285 & 838.813 \\
$D\left(10^{-9} \mathrm{~m}^{3} / \mathrm{s}^{2}\right)$ & 0.2923 & 0.3556 & 0.3646 & 0.4161 \\
$\gamma(\mathrm{mN} / \mathrm{m})$ & 23.0006 & 23.6031 & $\mathbf{2 6 . 2 5 3 6}$ & 25.1789 \\
\hline \hline
\end{tabular}

The structure factor $(S(Q))$ and potential energy surface were calculated to measure the effect of bond reduction of the molecule. The molecular structure was calculated from the X-ray structure factor $S(Q)$ in the reciprocal space $Q$. Fig. 1A shows the original and modified force field spectrum. The modified structure factors were not shifted; however, they were slightly modified from the original ones in parts of the spectrum. The $S(Q)$ is obtained by considering the combinations of the Fourier transform, $\hat{g}_{i} j(Q)$, for the radial distribution functions, $g_{i j}(r)$, where $i$ and $j$ run for all atom types in the system. In this way, we have

$$
S(Q)=\frac{\sum_{i=1}^{n} c_{i}^{2} f_{i}(Q) f_{i}(Q) \hat{g}_{i j}(Q)+\sum_{i \neq j}^{n} c_{i} c_{j} f_{i}(Q) f_{j}(Q) \hat{g}_{i j}(Q)}{\left(\sum_{i=1}^{n} f_{i}(Q)\right)^{2}}
$$

where $n$ is the number of atom species and $c_{i}$ are the molar fraction for each atom. The X-ray scattering form factors $f_{i}(Q)$ are defined by 


$$
f_{i}(Q)=\sum_{i=1}^{5} a_{i} e^{-b_{i} Q^{2}}+c
$$

where the parameters $a_{i}, b_{i}$, and $c$ are taken from [28].

On the other side, the energetic effect of the modified bond distance was measured by scanning the potential energy surface using quantum mechanics, which indicates that the potential energy was increasing, as seen in Fig. 1B, where it formed a parabola-like shape at distances close to the original bond $(1.529 \mathrm{~nm})$. This original bond distance was the lowest point of the curve, and the distance where the bond was reduced by $-2 \%$ had a difference of 1.05 $\mathrm{kJ} / \mathrm{mol}$ in the potential energy with an error of $0.0002 \%$ with respect to the original distance. This reduced distance was taken where the dielectric constant and surface tension has maximum values. Gaussian 09 package was used to obtain the potential energy with the theory b3lyp/6-311++g**.
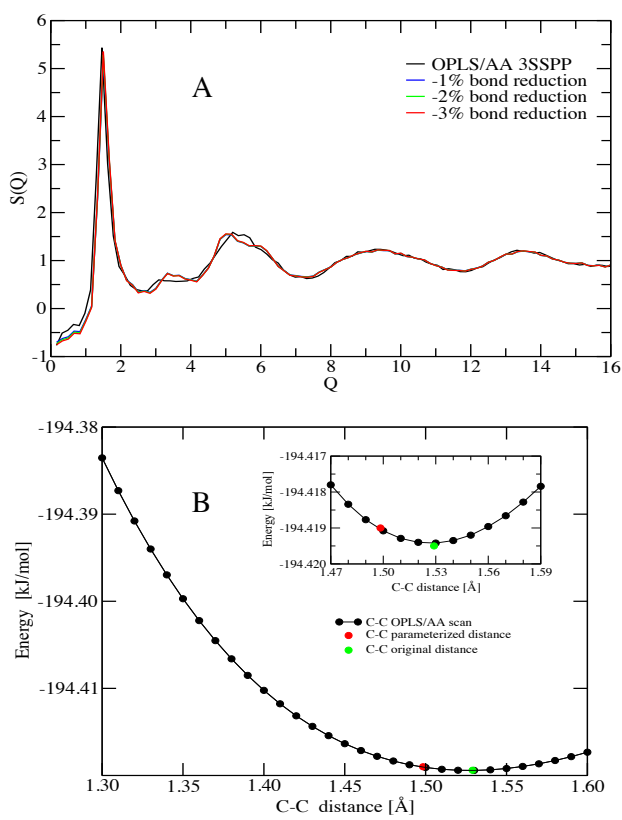

Fig. 1 A) Structure factor for 3SSPP AA 1-propanol for $0 \%, 1 \%, 2 \%$, and $3 \%$ bond distance reduction. B) Distance scan for AA 1-propanol molecule: comparing bonding distance and energy between original and parameterized force fields. 
In the UA force field, the modification of the force field was carried out by taking the parameterized force field [17] and applying a bond distance reduction to the molecule, a similar way to the AA force field; the maximum in dielectric constant and surface tension were obtained corresponding to $-2 \%$ reduction in the bond distance using this process. Moreover, Table 3 suggests that density and self-diffusion coefficient increased as the bond distance decreased.

Table 3 Dielectric constant, density, self-diffusion coefficient, and surface tension after $1 \%, 2 \%, 3 \%$, and $4 \%$ bond distance reduction of 1 -propanol with the parameterized UA force field. The maximum dielectric constant and surface tension are highlighted.

\begin{tabular}{crrrrr}
\hline \hline Property & UA 3SSPP & $-1 \%$ & $-2 \%$ & $-3 \%$ & $-4 \%$ \\
\hline \hline$\epsilon$ & 19.98 & 18.34 & $\mathbf{1 8 . 6 6}$ & 17.37 & 17.04 \\
$\rho\left(\mathrm{kg} / \mathrm{m}^{3}\right)$ & 797.39 & 805.549 & 813.173 & 820.379 & 828.149 \\
$D\left(10^{-9} \mathrm{~m}^{3} / \mathrm{s}^{2}\right)$ & 0.295 & 0.3088 & 0.3457 & 0.4323 & 0.4446 \\
$\gamma(\mathrm{mN} / \mathrm{m})$ & 23.3 & 26.9 & $\mathbf{2 7 . 3}$ & 26.9 & 28.9 \\
\hline \hline
\end{tabular}

\section{Results and discussions}

The effect of applying this new methodology in the AA and UA 1-propanol force field models can be deduced from Table 4, in which the estimated properties by reducing $2 \%$ of the bond distance for both force fields are listed. With the parameterized OPLS/AA force field, the dielectric constant, density, and surface tension exhibited percentage errors $<3 \%$ of all experimental data. For the self-diffusion coefficient, the simulational data was close to the lowest experimental value, with $<1 \%$ error. Using the same molecular dynamic parameters and a force field for UA, [17], we obtained the results tabulated in Table 4 , in which percentage errors $<3 \%$ were obtained using 3 SSPP/bond UA (column four) for almost all experimental data through the 3SSPP methodology [17]. The self-diffusion coefficient, was the only exception, being lower than the experimental data and slightly higher than the modified force field. 
Hence, the proposed procedure to parameterize the 1-propanol force field was applied to AA to reproduce the surface tension, density, dielectric constant, and self-diffusion coefficient.

Table 4 Estimated dielectric constant, density, self-diffusion coefficient, and surface tension from molecular simulations of 1-propanol in the OPLS/AA force field by applying 3SSPP/bond (column three) and for united atom model (column four). Percentage errors provided in parenthesis are concerned with experimental values. Experimental values are the same than Table 1.

\begin{tabular}{crr}
\hline \hline Property & 3SSPP/bond & 3SSPP/bond \\
& OPLS/AA \%error) & UA (\%error) \\
\hline \hline$\epsilon$ & $19.9(1.0)$ & $18.90(6.0)$ \\
& $(2.5)$ & $(7.4)$ \\
$\rho\left(k g / \mathrm{m}^{3}\right)$ & $793.2(0.9)$ & $799.939(0.0)$ \\
& $(1.3)$ & $(0.4)$ \\
$D\left(10^{-9} \mathrm{~m}^{3} / \mathrm{s}^{2}\right)$ & $0.556(0.7)$ & $0.3907(36.5)$ \\
& $(5.8)$ & $(40.6)$ \\
$\gamma(\mathrm{mN} / \mathrm{m})$ & $(11.3)$ & $(44.1)$ \\
\hline \hline
\end{tabular}

The original and new force field for AA and UA schemes are shown in the supporting information.

\subsection{Structure factor and bond distance}

In the AA model, the structure factors for pure substances in the original and the modified force fields were compared with the experimental spectrum obtained by X-ray diffraction [29], Fig. 2A. The difference between both simulations was imperceptible; the molecular structure was not affected by reducing the bond distance. An insignificant difference between the first maximum of both simulations and the experimental spectrum was the most meaningful change. For the UA model, depicted in Fig. 2B, we noted a similar behavior with respect to the original force field. The structure factor of the parameterized force field had fewer deviations from the experimental spectrum. 

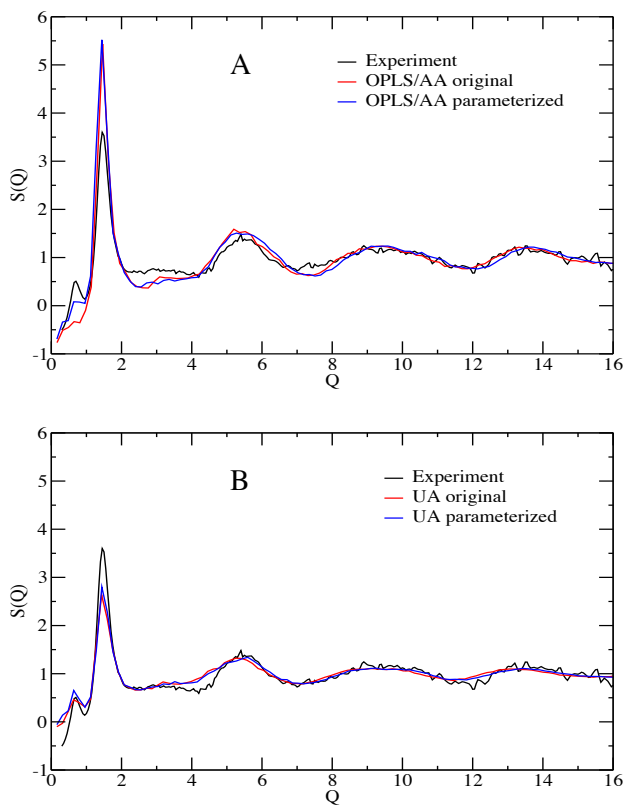

Fig. 2 Structure factor for A) AA and B) UA 1-propanol. The black line represents experimental conditions, the red and blue lines are the original and the 3SSPP/bond force field, respectively.

\subsection{Temperature dependence}

The temperature dependence of self-diffusion coefficient, density, and dielectric constant is shown in Figs. 3A, 3B, and 4, respectively. The diffusion behavior, shown in Fig. 3A, of the original AA model, is superdiffusive for all the experimental values (298.15 K, vertical black line). In the case of UA, the diffusion was close to the experimental values. In the new AA and UA force fields, the values were close to the experimental self-diffusion coefficient and their behavior at low temperatures was similar to that of the original one. The diffusion values obtained from AA were closer to the experimental value than those obtained from the UA modified force field. The UA (3SSPP/bond) obtained self-diffusion coefficients greater than the 3SSPP UA force field (Tables 1 and $4)$. 

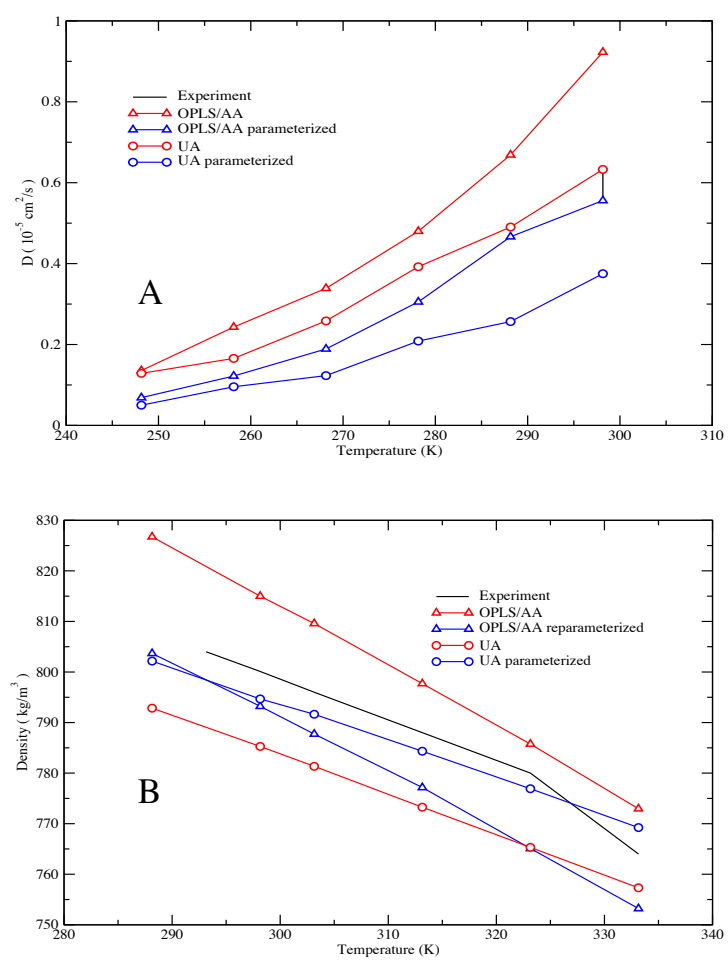

Fig. 3 Temperature dependence of A) self-diffusion coefficient and B) density obtained using AA and UA models in the 1-propanol force field. The black line is the experimental data, the circles and triangles correspond to the data obtained from the UA and AA models, respectively. The blue and red lines correspond to the values obtained from the $3 \mathrm{SSPP} /$ bond and original force field, respectively.

The density was obtained by adjusting the force fields to the experimental values, with low errors (around $2 \%$ or less). Fig. 3B shows that the results obtained from all the force fields are close to the experimental values; the obtained results are more accurate than the original ones for the AA and UA parameterized force fields. The data display a similar constant slope after parameterizing the force fields.

Low dielectric constant and/or super self-diffusion coefficients are the common characteristics across all force fields. In Fig. 4, the dielectric constant in the new force field is greater than that in the original force field, whereas 
the self-diffusion coefficients were higher for both the AA and UA models, particularly the AA force field.

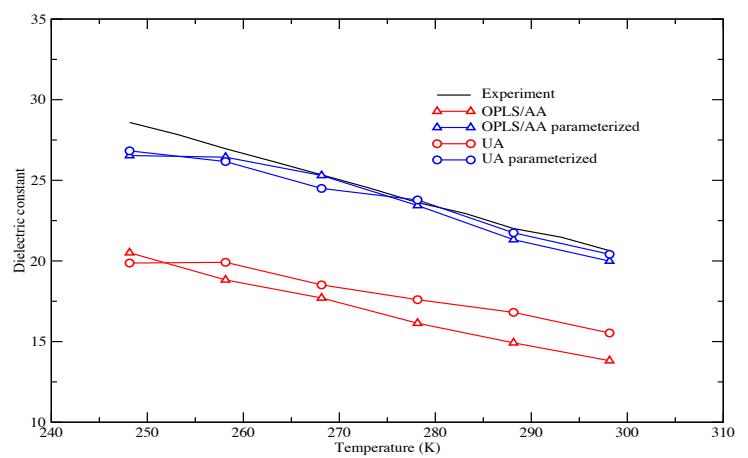

Fig. 4 Dependence of dielectric constant on temperature obtained using AA and UA model in the 1-propanol force field. The black line reflects the experimental data, the circles represent the UA model and the triangles correspond to the AA model. The blue and red lines represent the 3SSPP/bond and original force field, respectively.

\subsection{Solubility}

The solubility was calculated for the original and parameterized force fields. The solubility was used to parameterize force fields and was related with charge distribution [30]. The water models used are SPCE [31] and TIP4P/ $\epsilon$ [10]; both are the best models that fit basic experimental properties (Supplementary Information). The 1-propanol experimental solubility in water is $1000 \mathrm{~g} / \mathrm{L}$.

The solubility of a solute $i$ in solvent $j$ was obtained from the density profiles,

$$
S_{i}=\frac{\rho_{i}(z)}{M_{i} \rho_{j}(z)}
$$

where, $M_{i}$ is the solute molar mass in $\mathrm{kg} / \mathrm{mol}, \rho_{i}(z)$ and $\rho_{j}(z)$ are the density profiles for the solute and solvent, respectively. 
Fig 5 shows the density profiles of parameterized and original AA 1propanol force fields in water. The mixture of 1-propanol with SPCE is shown in Fig. 5A and for TIP4P/ $\epsilon$ in Fig. 5B. The Fig. 5A shows complete miscibility before modifying the force field and it is maintained after applying the 3SSP$\mathrm{P} /$ bond methodology. On the other hand, Fig. 5B shows a similar behavior that concludes that AA model is soluble for both water models.

On the contrary, Fig. 6A shows density profiles of parameterized and original UA 1-propanol force fields and SPCE water. The functions oscillated indicating partial miscibility. For the original 1-propanol, the solubility was $194.2 \mathrm{~g} / \mathrm{L}$; after parameterizing the force field, this value increased to 322.4 $\mathrm{g} / \mathrm{L}$. The solubility was improved by considering the TIP4P/ $\epsilon$ water model (Fig. 6B) where the value for solubility before applying the 3SSPP/bond methodology was $280 \mathrm{~g} / \mathrm{L}$, it is greater than that with the SPCE water model. However, in the parameterized scheme, the 1-propanol is miscible in TIP4P/ $\epsilon$ water model.

\section{Conclusions}

In this work, we studied and proposed a new force field for 1-propanol AA and UA schemes by applying a new methodology called 3SSPP/bond. We noted that the proposed parameterization method provided consistent simulation results with experimental findings for AA with minimal error pertaining to the dielectric constant, density, surface tension, and self-diffusion coefficient. For the UA model, the self-diffusion coefficient was lower than the experimental value but increased with the 3SSPP methodology.

The structure factor for the new force field used in both molecular models did not change significantly with reduction in bond distance and the difference in bond potential energy was not modified significantly; these configurations 

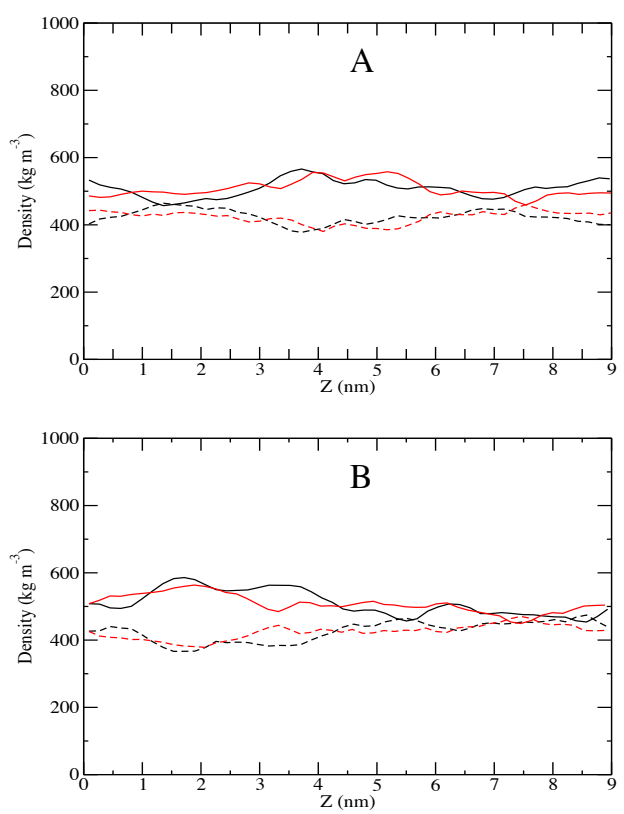

Fig. 5 Density profiles were obtained from the AA force field in water, for A) SPCE and B) TIP4P/ $/ \epsilon$. The solid and the dashed lines represent the density profiles for water and 1propanol, respectively. The black and red lines correspond to the data obtained using the original and 3SSPP/bond force fields, respectively.

indicated that this parametrized model was suitable to parameterize the 1propanol force field.

The temperature dependence of density and dielectric constant, fitted the experimental data using the AA and UA models. The temperature dependence of the self-diffusion coefficient exhibited the right behavior for the temperature range obtained for both atomistic 1-propanol models.

In the case of solubility, the AA force field was water-soluble before applying the 3SSPP/bond methodology. This solubility remained constant after parameterizing by using two different water models, TIP4P/ $\epsilon$ and SPCE. For the UA model, the obtained solubility was low for the original force field; the parameterization increased the solubility and for TIP4P/ $\epsilon$ water model this property reached the experimental value. 

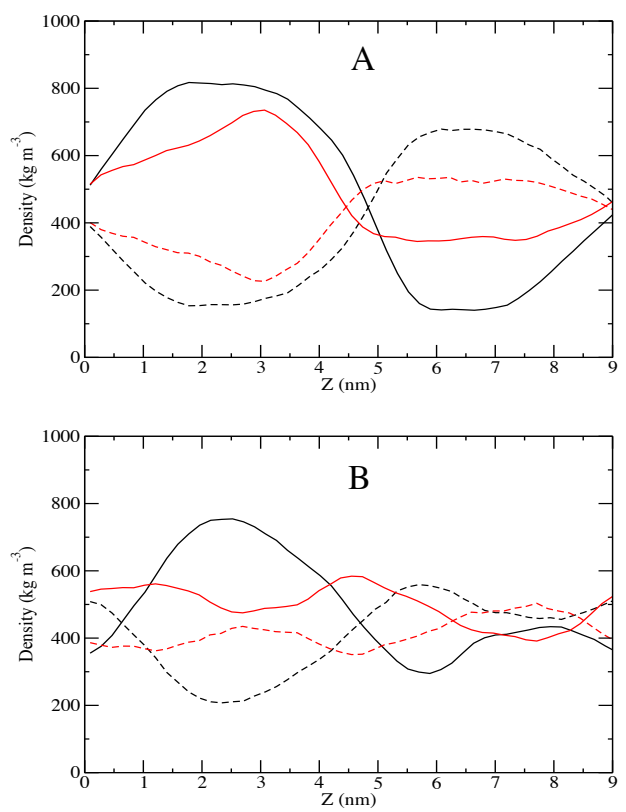

Fig. 6 Density profiles were obtained from the UA force field in water, for A) SPCE and B) TIP4P/ $/ \epsilon$. The solid and dashed lines indicated the density profile for water and the 1propanol, respectively. The black and the red lines correspond to the data obtained using the original and 3SSPP/bond force fields, respectively.

Supplementary information. The Supplementary Information is available free of charge at: https://wwww.

Original and parametrized force field for AA and UA schemes. Properties for some water models used to calculate solubility.

Acknowledgments. The authors gratefully acknowledge the supercomputer facilities of Laboratorio Nacional de Supercómputo del Suroeste de México (LNS) project 201801014N1R and 201901004N. IGE, GAMM, and JGMB acknowledge PRODEP. JGMB acknowledges SNI-CONACyT. This work was supported by grant CB-2016-01 286238 from CONACyT, México. 


\section{Declarations}

- Funding. The authors would like to acknowledge the partial financial support of CONACyT-México (Grant No. CB-2016-01 286238). José Guillermo Méndez Bermúdez acknowledges Sistema Nacional de Investigadores, CONACyT-México.

- Conflict of interest/Competing interests. The authors declare no conflict/competing interests.

- Availability of data. All generated data for this study are avialable along with this manusscript.

- Code availability. All molecular dynamics' data are obtained with GROMACS code. Potential energy surface was calculated with Gaussian software. Figures are made in XMGRACE grapher.

- Authors' contributions. All authors contribuited equally to this work; José Abundio Daniel Alva-Tamayo: Reviwing, Editing; José Guillermo MéndezBermúdez: Writing, Reviwing, Editing.

\section{References}

[1] Jorgensen WL, Tirado-Rives J (2005) Potential energy functions for atomic-level simulations of water and organic and biomolecular systems. Proc. Natl. Acad. Sci. USA 102:6665-6670. Dodda LS, Vilseck JZ, Tirado-Rives J, Jorgensen WL (2017) 1.14*CM1A-LBCC: Localized BondCharge Corrected CM1A Charges for Condensed-Phase Simulations. J. Phys. Chem. B 121:3864-3870. Dodda LS, Cabeza de Vaca I, TiradoRives J, Jorgensen WL (2017) LigParGen web server: An automatic OPLS-AA parameter generator for organic ligands. Nucleic Acids Res. 45:W331-W336. 
[2] Oostenbrink C, Villa A, Mark AE, van Gunsteren WF (2004) A Biomolecular Force Field Based on the Free Enthalpy of Hydration and Solvation: The GROMOS Force-Field Parameter Sets 53A5 and 53A6. J. Comput. Chem. 25:1656-1676.

[3] Brooks BR, Bruccoleri RE, Olafson BD, States DJ, Swaminathan S, Karplus M (1983) CHARMM: A Program for Macromolecular Energy, Minimization and Dynamics Calculations. J. Comput. Chem. 4:187-217.

[4] Wang J, Wolf RM, Caldwell JW, Kollman PA, Case DA (2004) Development and testing of a general amber force field. J. Comput. Chem. 25:1157-1174.

[5] Cole DJ, Vilseck JZ, Tirado-Rives J, Payne JMC, Jorgensen WL (2016) Biomolecular force field parameterization via Atoms-in-Molecules Electron Density Partitioning. J. Chem. Theory Comput. 12:2312-2323.

[6] Caleman C, van Maaren CPJ, Hong M, Hub JS, Costa LT, van der Spoel D (2012) Force Field Benchmark of Organic Liquids: Density, Enthalpy of Vaporization, Heat Capacities, SurfaceTension, Isothermal Compressibility, Volumetric Expansion Coefficient, and Dielectric Constant. J. Chem. Theory Comput. 8:61-74.

[7] Salas FJ, Méndez-Maldonado GA, Núñez-Rojas E, Aguilar-Pineda GE, Domínguez H, Alejandre J (2015) Systematic Procedure To Parametrize Force Fields for Molecular Fluids. J. Chem. Theory Comput. 11:683-693.

[8] García-Melgarejo V, Núñez-Rojas E, Alejandre J (2021) United atom model via interactions with explicit water (UAMI-EW): Alcohols and ketones. J. Molecular Liquids 323:114576.

[9] Fuentes-Azcatl R, Mendoza N, Alejandre J (2015) Improved SPC force field of water based on the dielectric constant: SPC/ $\epsilon$. Phys. A 420:116-123.

[10] Fuentes-Azcatl R, Alejandre J (2014) Non-Polarizable Force Field of 
Water Based on the Dielectric Constant: TIP4P/€. J. Phys. Chem. B 118:1263-1272.

[11] Ríos-López M, Mendez-Bermúdez JG, Dominguez H (2018) New Force Field Parameters for Sodium Dodecyl Sulfate and Olefin Sulfonate Anionic Surfactants. J. Phys. Chem B 122:4558-4565. Ríos-López M, MendezBermúdez JG, Vásquez-Sánchez MIV, Dominguez H (2019) Surface tension calculations of the cationic (CTAB) and the zwitterionic (SB3-12) surfactants using new force field models: A computational study. Mol. Phys. 117:3632-3641.

[12] Fuentes-Azcatl R, Domínguez H (2019) Prediction of experimental properties of $\mathrm{CO}_{2}$ : improving actual force fields. J. Mol. Model 25:146.

[13] Harris JG, Yung KH (1995) Carbon Dioxide Liquid-Vapor Coexistence Curve and Critical Properties As Predicted by a Simple Molecular Model. J. Phys. Chem. 99:12021-12024.

[14] Pérez de la Luz A, Aguilar-Pineda JA, Méndez-Bermúdez JG, Alejandre J (2018) Force Field Parameterization from the Hirshfeld Molecular Electronic Density. J. Chem. Theory Comput. 14:5949-5958.

[15] Martínez-Jiménez M, Saint-Martin H (2018) A Four-Site Molecular Model for Simulations of Liquid Methanol and Water-Methanol Mixtures: MeOH4p. J. Chem Theory Comput. 14:2526-2537.

[16] Kulschewski T, Pleiss J (2013) A Molecular Dynamics Study of Liquid Aliphatic Alcohols: Simulation of Density and Self-Diffusion Coeffcient Using a Modified OPLS Force Field. Mol. Sim. 39:754-767.

[17] Méndez-Bermúdez JG, Dominguez H, Pustai L, Guba S, Horváth B, Szalai I (2016) Composition and temperature dependence of the dielectric of 1-propanol/water mixtures: Experiment and molecular dynamics simulations. J. Mol. Liq. 219:354-358. 
[18] Zangi R, (2018) Refinement of the OPLSAA Force-Field for Liquid Alcohols. ACS Omega 3:18089-18099.

[19] Allen MP, Tildesley DJ (2017) Computer simulation of liquids. 2nd Edn. Oxford University Press, Oxford. Frenkel D, Smit B (2001) Understanding Molecular Simulation: from algorithms to applications, 2nd Edn. Academic Press, Amsterdam.

[20] Abraham MJ, Murtola T, Schulz R, Páll S, Smith JC, Hess B, Lindahl E (2015) GROMACS: High performance molecular simulations through multi-level parallelism from laptops to supercomputers. Software X, 1-2: $19-25$.

[21] Maryott AA, Smith ER (1951) Table of dielectric constants of pure liquids, Natl. Circ. No. 514, Bur. Stand. U.S. GPO, Washington, D.C.

[22] Pang F-M, Seng C-E, Teng T-T, Ibrahim MH (2017) Densities and viscosities of aqueous solutions of 1-propanol and 2-propanol at temperatures from 293.15 K to 333.15 K. J. Mol. Liq. 136:71-78.

[23] Haynes WM (2010) CRC Handbook of Chemistry and Physics. CRC Press/Taylor and Francis, Boca Raton, FL.

[24] McCall DW, Douglass DC (1960) Self-diffusion in the primary alcohols, J. Chem. Phys. 32:1876-1877.

[25] Pratt KC, Wakeham WA (1977) Self-diffusion in water and monohydric alcohols. J. Chem. Soc., Faraday Trans. 2: Mol. Chem. Phys. 73:997-1002.

[26] Tofts P.S, Lloyd D, Clark CA, Barker GJ, Parker GJM, McConville P, Baldock C, J. Pope M (2000) Test liquids for quantitative MRI measurements of self-diffusion coefficient in vivo. Magn. Reson. Med. 43:368-374.

[27] Vazquez G, Alvarez E, Navaza JM (1995) Surface Tension of Alcohols + Water from 20 to $50{ }^{\circ}$ C. J. Chem. Eng. Data 40:611-614.

[28] Waasmaier D, Kirfel A (1995) New analytical scattering-factor functions 
for free atoms and ions. Acta Cryst. A51:416-431.

[29] Méndez-Bermúdez JG, Dominguez H, Temleitner L, Pusztai L (2018) On the Structure Factors of the Aqueous Mixtures of 1-Propanol and 2-Propanol: X-Ray Diffraction Experiments and Molecular Dynamics Simulations. Phys. Status solidi B 255:1800215.

[30] Núñez-Rojas E, García-Melgarejo V, Pérez de la Luz A, Alejandre J (2019) Systematic parameterization procedure to develop force fields for molecular fluids using explicit water. Fluid Phase Equilib. 490:1-12.

[31] Berendsen HJC, Grigera JR, Straatsma TP (1987) The missing term in effective pair potentials. J. Phys. Chem. 91:6269-6271. 


\title{
Supplementary information for: Improving 1-propanol force field: a new methodolgy
}

\author{
José Abundio Daniel Alva-Tamayo ${ }^{\dagger}$, Iván Guillén-Escamilla†, \\ Gloria Arlette Méndez-Maldonado ${ }^{\ddagger}$ José Guillermo Méndez-Bermúdez $^{\dagger} *$ \\ $\dagger$ Centro Universitario de los Valles, Universidad de Guadalajara, \\ Carretera Guadalajara-Ameca Km. 45.5, Ameca 46600, Jalisco, México. \\ $\ddagger$ Centro Universitario de Ciencias Exactas e Ingenierías, Universidad de Guadalajara \\ Blvd. Marcelino García Barragán No. 1412, Guadalajara 44430, Jalisco, México.
}

\section{Force Fields parameters}

A) OPLS/AA parameters for the original and 3SSPP/bond force fields.

\begin{tabular}{lrccc}
\hline \hline Atom & $\begin{array}{r}\sigma(\mathrm{nm}) \\
\text { Original }\end{array}$ & $\begin{array}{c}\sigma(\mathrm{nm}) \\
3 S S P P / \text { bond }\end{array}$ & $\begin{array}{c}\epsilon(\mathrm{kJ} / \mathrm{mol}) \\
\text { Original }\end{array}$ & $\begin{array}{c}\epsilon(\mathrm{kJ} / \mathrm{mol}) \\
3 S S P P / \text { bond }\end{array}$ \\
\hline $\mathrm{H}(\mathrm{C})$ & 0.250 & 0.257550 & 0.125520 & 0.101671 \\
$\mathrm{C}$ & 0.350 & 0.360570 & 0.276144 & 0.223677 \\
$\mathrm{O}(\mathrm{H})$ & 0.312 & 0.321422 & 0.711280 & 0.576137 \\
$\mathrm{H}(\mathrm{O})$ & 0.000 & 0.000000 & 0.000000 & 0.000000 \\
\hline \hline Bond & $\mathrm{r}_{o}(\mathrm{~nm})$ & $\mathrm{r}_{o}(\mathrm{~nm})$ & & \\
& Original & 3 SSPP $/$ bond & & \\
\hline $\mathrm{H}-\mathrm{C}$ & 0.1090 & 0.10682 & & \\
$\mathrm{C}-\mathrm{C}$ & 0.1529 & 0.14984 & & \\
$\mathrm{C}-\mathrm{O}$ & 0.1410 & 0.13818 & & \\
$\mathrm{O}-\mathrm{H}$ & 0.0945 & 0.09261 & & \\
\hline \hline $\mathrm{Charge}$ & Original & $3 \mathrm{SSPP} /$ bond & & \\
\hline $\mathrm{C}\left(\mathrm{CH}{ }_{3}\right)$ & -0.2359 & -0.2689 & & \\
$\mathrm{C}\left(\mathrm{CH}{ }_{2}\right)$ & -0.2257 & -0.2573 & & \\
$\mathrm{C}(\mathrm{O})$ & 0.1102 & 0.1256 & & \\
$\mathrm{H}\left(\mathrm{CH}{ }_{3}\right)$ & 0.0841 & 0.0959 & & \\
$\mathrm{H}\left(\mathrm{CH}{ }_{2}\right)$ & 0.0958 & 0.1092 & & \\
$\mathrm{H}(\mathrm{CO})$ & 00951. & 0.1084 & & \\
$\mathrm{O}(\mathrm{H})$ & -0.6883 & -0.7847 & & \\
$\mathrm{H}(\mathrm{O})$ & 0.4056 & 0.4624 & & \\
\hline \hline
\end{tabular}

Table 1: Charge, bond distance, $\sigma$ and $\epsilon$ Lennard-Jones parameters. The original parameters were taken of [1]

\footnotetext{
*Author to whom correspondence should be addressed. jose.bermudez@academicos.udg.mx
} 


\section{B) UA parameters for original UA [2] and 3SSPP/bond force field.}

\begin{tabular}{lrccc}
\hline \hline Atom & $\begin{array}{r}\sigma(\mathrm{nm}) \\
\text { Original }\end{array}$ & $\begin{array}{c}\sigma(\mathrm{nm}) \\
\text { 3SSPP } / \text { bond }\end{array}$ & $\begin{array}{c}\epsilon(\mathrm{kJ} / \mathrm{mol}) \\
\text { Original }\end{array}$ & $\begin{array}{c}\epsilon(\mathrm{kJ} / \mathrm{mol}) \\
3 \mathrm{SSPP} / \text { bond }\end{array}$ \\
\hline $\mathrm{C}\left(\mathrm{CH}_{3}\right)$ & 0.391 & 0.39296 & 0.899288 & 0.836348 \\
$\mathrm{C}\left(\mathrm{CH}_{2}\right)$ & 0.393 & 0.39497 & 0.396932 & 0.368310 \\
$\mathrm{C}(\mathrm{O})$ & 0.393 & 0.39497 & 0.396932 & 0.368310 \\
$\mathrm{O}(\mathrm{H})$ & 0.298 & 0.29949 & 0.933816 & 0.868449 \\
$\mathrm{H}(\mathrm{O})$ & 0.098 & 0.09849 & 0.033696 & 0.031332 \\
\hline \hline Bond & $\mathrm{r}_{o}(\mathrm{~nm})$ & $\mathrm{r}_{o}(\mathrm{~nm})$ & & \\
& Original & 3 SSPP $/$ bond & & \\
\hline $\mathrm{C}-\mathrm{C}$ & 0.1540 & 0.15092 & & \\
$\mathrm{C}-\mathrm{O}$ & 0.1428 & 0.13994 & & \\
$\mathrm{O}-\mathrm{H}$ & 0.0961 & 0.09418 & & \\
\hline \hline $\mathrm{Charge}$ & Original & 3 SSPP $/$ bond & & \\
\hline $\mathrm{C}\left(\mathrm{CH}_{3}\right)$ & 0.0000 & 0.0000 & & \\
$\mathrm{C}\left(\mathrm{CH}_{2}\right)$ & 0.0000 & 0.0000 & & \\
$\mathrm{C}(\mathrm{O})$ & 0.2900 & 0.3196 & & \\
$\mathrm{O}(\mathrm{H})$ & -0.7100 & -0.7825 & 0.4629 & \\
$\mathrm{H}(\mathrm{O})$ & 0.4200 & & & \\
\hline \hline
\end{tabular}

Table 2: Charge, bond distance and the $\sigma, \epsilon$ Lennard-Jones parameters. The original UA parameters were taken of [2].

\section{Solubility: water models}

Different properties are calculated for some water models to obtain solubility. These properties are: self-diffusion coefficient vs hydrogen bonds, surface tension vs dielectric constant and density in two different combination rules; lorentz-Bertelot (combination rule 2) and geometric average for Lennard-Jones parameters (combination rule 3). These water models are: TIP3P[4], SPCE[5], TIP4P $/ 2005$ [6], $\mathrm{TIP} 4 \mathrm{P} / \epsilon[7], \mathrm{SPCE} / \epsilon[8]$. We note that, $\mathrm{SPCE}$ (3-site type) and TIP4P/ $/ \epsilon$ (4-site type) models are close to the experimental properties mentioned above. All molecular simulations were made in Gromacs software [3]. 

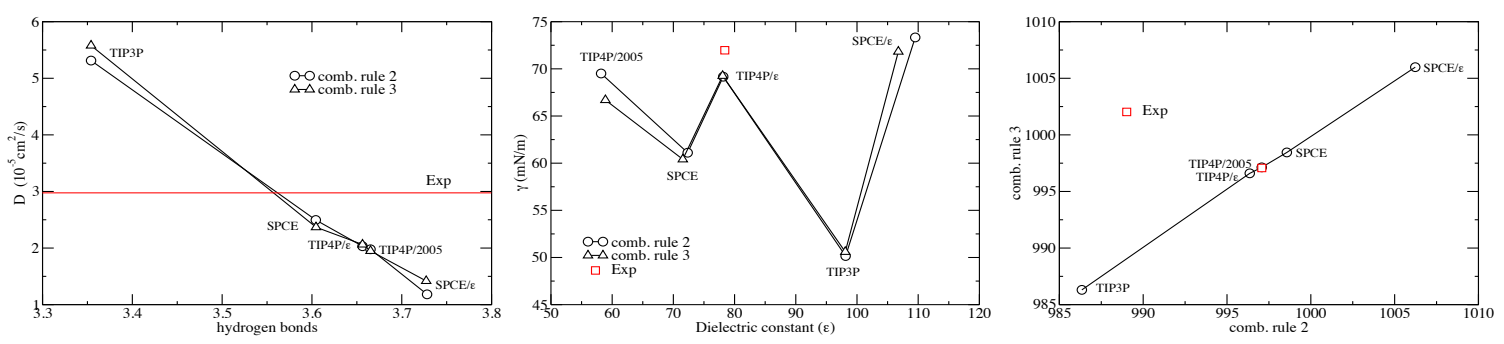

Figure 1: Water properties of different water models. Left figure, self-diffusion coefficient vs hydrogen bonds, the red line represent the experimental value. Center figure, surface tension vs dielectric constant, the red square is the experimental data. Right figure, density in two different combination rules; Lorentz-Berthelot (combination rule 2) and geometric average for Lennard-Jones parameters (combination rule $3)$, the red square is the experimental value.

\section{References}

[1] Jorgensen WL, Tirado-Rives J (2005) Potential energy functions for atomiclevel simulations of water and organic and biomolecular systems. Proc. Natl. Acad. Sci. USA 102:6665-6670. Dodda LS, Vilseck JZ, Tirado-Rives J, Jorgensen WL (2017) 1.14*CM1A-LBCC: Localized Bond-Charge Corrected CM1A Charges for Condensed-Phase Simulations. J. Phys. Chem. B 121:3864-3870. Dodda LS, Cabeza de Vaca I, Tirado-Rives J, Jorgensen WL (2017) LigParGen web server: An automatic OPLS-AA parameter generator for organic ligands. Nucleic Acids Research 45:W331-W336.

[2] Khare R, Sum AK. Nath SK, de Pablo JJ (2004) Simulation of Vapor-Liquid Phase Equilibria of Primary Alcohols and Alcohol-alkane Mixtures. J. Phys. Chem. B 108:10071-10076.

[3] Abraham MJ, Murtola T, Schulz R, Páll S, Smith JC, Hess B, Lindahl E (2015) GROMACS: High performance molecular simulations through multi-level parallelism from laptops to supercomputers. Software X, 1-2:19-25.

[4] Jorgensen WL, Chandrasekhar J, Madura JD, Impey RW, Klein ML (1983) Comparation of simple potentila functions for simulating liquid water. J. Chem. Phys. 79:926-935.

[5] Berendsen HJC, Grigera JR, Straatsma TP (1987) The missing term in effective pair potentials. J. Phys. Chem. 91:6269-6271.

[6] Abascal JLF, Vega C (2005) A general purpose model for the condensed phases of water:TIP4P/2005. J. Chem. Phys. 123:234505. 
[7] Fuentes-Azcatl R, Alejandre J (2014) Non-Polarizable Force Field of Water Based on the Dielectric Constant:TIP4P/ $\epsilon$. J. Phys. Chem. B 118:1263-1272.

[8] Fuentes-Azcatl R, Mendoza N, Alejandre J (2015) Improved SPC force field of water based on the dielectric constant: SPC/ $\epsilon$. Phys. A 420:116-123. 


\section{Supplementary Files}

This is a list of supplementary files associated with this preprint. Click to download.

- GA1.eps 